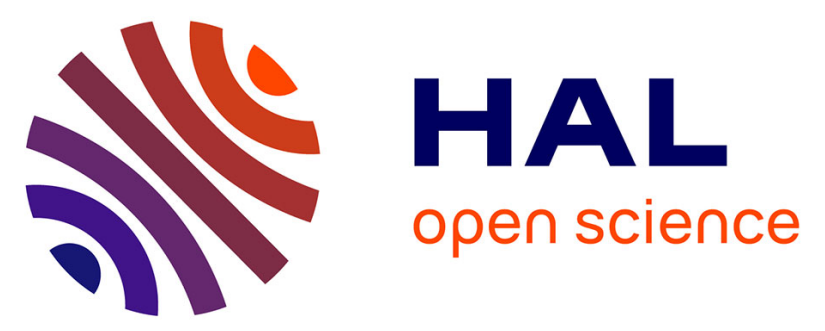

\title{
Dépôts carbonatés et fonctionnement des aqueducs romains: le bassin amont du vallon des Arcs sur l'aqueduc d'Arles (Bouches-du-Rhône)
}

Jean-Louis Guendon, Philippe Leveau

\section{- To cite this version:}

Jean-Louis Guendon, Philippe Leveau. Dépôts carbonatés et fonctionnement des aqueducs romains : le bassin amont du vallon des Arcs sur l'aqueduc d'Arles (Bouches-du-Rhône). Gallia - Archéologie de la France antique, 2005, Aqueducs de la Gaule méditerranéenne, 62, pp.87-96. 10.3406/galia.2005.3222 . hal-01912619

\section{HAL Id: hal-01912619 \\ https://hal.science/hal-01912619}

Submitted on 14 Jan 2020

HAL is a multi-disciplinary open access archive for the deposit and dissemination of scientific research documents, whether they are published or not. The documents may come from teaching and research institutions in France or abroad, or from public or private research centers.
L'archive ouverte pluridisciplinaire HAL, est destinée au dépôt et à la diffusion de documents scientifiques de niveau recherche, publiés ou non, émanant des établissements d'enseignement et de recherche français ou étrangers, des laboratoires publics ou privés.

\section{(1) (1) $\$$}

Distributed under a Creative Commons Attribution - NonCommercial - NoDerivatives 44.0 


\title{
DÉPÔTS CARBONATÉS ET FONCTIONNEMENT DES AQUEDUCS ROMAINS
}

\author{
Le bassin amont $d u$ vallon des Arcs \\ sur l'aqueduc d'Arles (Bouches-du-Rhône)
}

Jean-Louis GUENDON avec la collaboration de Philippe LEVEAU

Mots-clés. Sud-est de la France, aqueducs romains, concrétions calcaires, travertins.

Résumé. L'étude des concrétions du bassin de convergence du vallon des Arcs a permis de préciser l'histoire des différents aménagements de cette partie de l'aqueduc antique d'Arles et a fourni des arguments décisifs pour dater la construction des moulins de Barbegal.

Key-words. South-Eastern France, Roman aqueducts, calcareous concretions, travertines.

Abstract. The study of concretions of the Roman aqueduct of Arles has provided unambiguous informations for the reconstruction of the various arrangements of this part of the aqueduct. It enables to tell when the Barbegal flour-mills were built.

Translation : Isabelle FALDLET

Schlagwörter. Südostfrankreich, römische Wasserleitung, Kalkablagerungen, Travertin.

Zusammenfassung. Eine Untersuchung der Kalkablagerungen des Sammelbeckens von vallon des Arcs ermöglicht es, den Ablauf der veschiedenen Umbaumaßnahmen dieses Abschnitts der antiken Wasserleitung von Arles genauer zu beschreiben und liefert entscheidende Argumente für die Datierung der römischen Mühlen von Barbegal.

Übersetzung : Stefan WIRTH

Dans l'Antiquité, comme de nos jours, les grandes sources captées pour l'alimentation des villes sont très souvent issues des massifs karstiques (constitués essentiellement de roches calcaires ou dolomitiques), surtout sur le pourtour méditerranéerı où abondent de tels massifs. C'est le cas des principaux aqueducs de la Gaule méditerranéenne: Fréjus, Aix-enProvence, Arles, Nîmes, Béziers, etc. Ces eaux karstiques sont riches en bicarbonate de calcium dissout. De ce fait, elles ont déposé au cours du temps des concrétions, des incrustations calcaires plus ou moins importantes sur les parois des conduits. Ces dépôts, source de problèmes à l'époque (entrave à la circulation de l'eau), s'avèrent aujourd'hui riches en informations pour l'étude de l'histoire des ouvrages hydrauliques et apparaissent comme de fidèles témoins du fonctionnement d'un aqueduc.

Plusieurs études leur ont déjà été consacrées.

J.-P. Adolphe s'intéresse aux concrétions du Pont du Gard dans ses travaux sur la genèse des encroûtements carbonatés et le rôle des processus biochimiques dans la précipitation des carbonates (Adolphe, 1973, 1981). M. Bailhache fait intervenir les dépôts calcaires dans ses calculs de débits pour quelques aqueducs gallo-romains. Il fait ressortir le rôle néfaste de celles- ci dans l'écoulement des eaux et note que leur examen "permet d'apprécier la "vie" de l'aqueduc " (Bailhache, 1979, 1983). P. Verrez et surtout J.-C. Gilly, à travers l'analyse chimique des dépôts de l'aqueduc de Nîmes, se servent des concrétions comme indicateurs paléoclimatologiques et paléohydrologiques (Gilly et al., 1971, 1978 ; Verrez, 1985). Enfin, dans l'atlas de l'aqueduc romain de Cologne (Grewe, 1986), deux contributions sont consacrées aux concrétions, avec pour thèmes essentiels les paléodébits, leur genèse (Brinker, 1986) et leur utilisation pour la datation relative (Schulz, 1986).

Les travaux récents sur différents aqueducs gallo-romains du Midi de la France (Nîmes, Arles, Fréjus) ont montré tout l'intérêt que pouvait présenter l'étude détaillée de ces incrustations pour la recherche archéologique et la diversité des informations qu'elles peuvent apporter dans ce domaine (Guendon, Vaudour, 1986, 2000 ; Guendon, Villeminot, 1992 ; Guendon et al., 1994, 2002 ; Bonté, 1999 ; Blanc, 2000 ; Joseph et al., 2000 ; Bobée, 2002). Dans le cas de l'aqueduc d'Arles l'attention s'est essentiellement portée sur le bassin de convergence à l'amont du pont-aqueduc du vallon des Arcs. Les concrétionnements ont, en effet, enregistré les utilisations successives d'un bassin dont la destination initiale était 
d'assurer la convergence des deux branches de l'aqueduc d'Arles (fig. 79).

\section{LES AQUEDUCS D'ARLES ET DE BARBEGAL}

\section{DESCRIPTION SOMMAIRE DE L'ENSEMBLE DE L'OUVRAGE}

Dans ce dossier, le double système des ponts-aqueducs franchissant le vallon des Arcs à l'amont des moulins de Barbegal fait l'objet d'une étude architecturale qui en décrit la construction et les remaniements successifs (voir infra Leveau et Thernot, p. 97-105). L'histoire complexe de ces ouvrages s'explique d'abord par la double destination de l'eau circulant dans les canaux que supportaient ces architectures: la ville d'Arles, premier et principal destinataire; les moulins construits en contrebas du chaînon de la Pène, à Barbegal. Elle s'explique aussi par la double origine de l'eau. Les fouilles archéologiques dirigées par P. Leveau (de 1995 à 2000) ont, en effet, montré qu'avant la construction des moulins les deux aqueducs correspondant à ces deux utilisations, dont le tracé était connu depuis le XIX ${ }^{\mathrm{c}} \mathrm{s}$. (Grenier, 1960), avaient convergé dans un bassin situé à l'amont des ponts.

\section{DESCRIPTION DU SITE DU VALLON DES ARCS}

De l'étude archéologique du bassin et des conduits (Leveau, 1995), nous retiendrons les points essentiels suivants. Le bassin où convergent les deux aqueducs a une forme rectangulaire et une profondeur de $1,50 \mathrm{~m}$ (fig. 80 et 81 ). Au nord et à l'est, il reçoit deux conduits d'alimentation. Nous appelons l'un « aqueduc nord " ou " de Saint Rémy ", car il provient du flanc nord des Alpilles et l'autre " aqueduc sud » ou " des Baux ", car il draine les eaux du versant sud du même massif (fig. 79). Dans la partie sud du bassin, s'échappe un troisième conduit qui alimentait directement Arles (canal d'Arles). Une quatrième ouverture, anciennement murée, puis rouverte dans sa partie supérieure pour ménager un petit déversoir, existe sur le flanc ouest du bassin : ouverture murée occidentale. Six mètres avant de rejoindre le bassin de convergence, l'aqueduc des Baux présente un canal diffluent vers le sud (canal des moulins), qui conduisait les eaux directement à la meunerie de Barbegal. Deux monolithes arment le départ de ce canal. On appelle bras mort le tronçon de l'aqueduc des Baux compris entre cette diffluence et le bassin de convergence, car il a été obturé pendant un certain temps :

- à son extrémité amont (est), un mur a été construit à un moment donné pour fermer le canal, puis, dans une phase

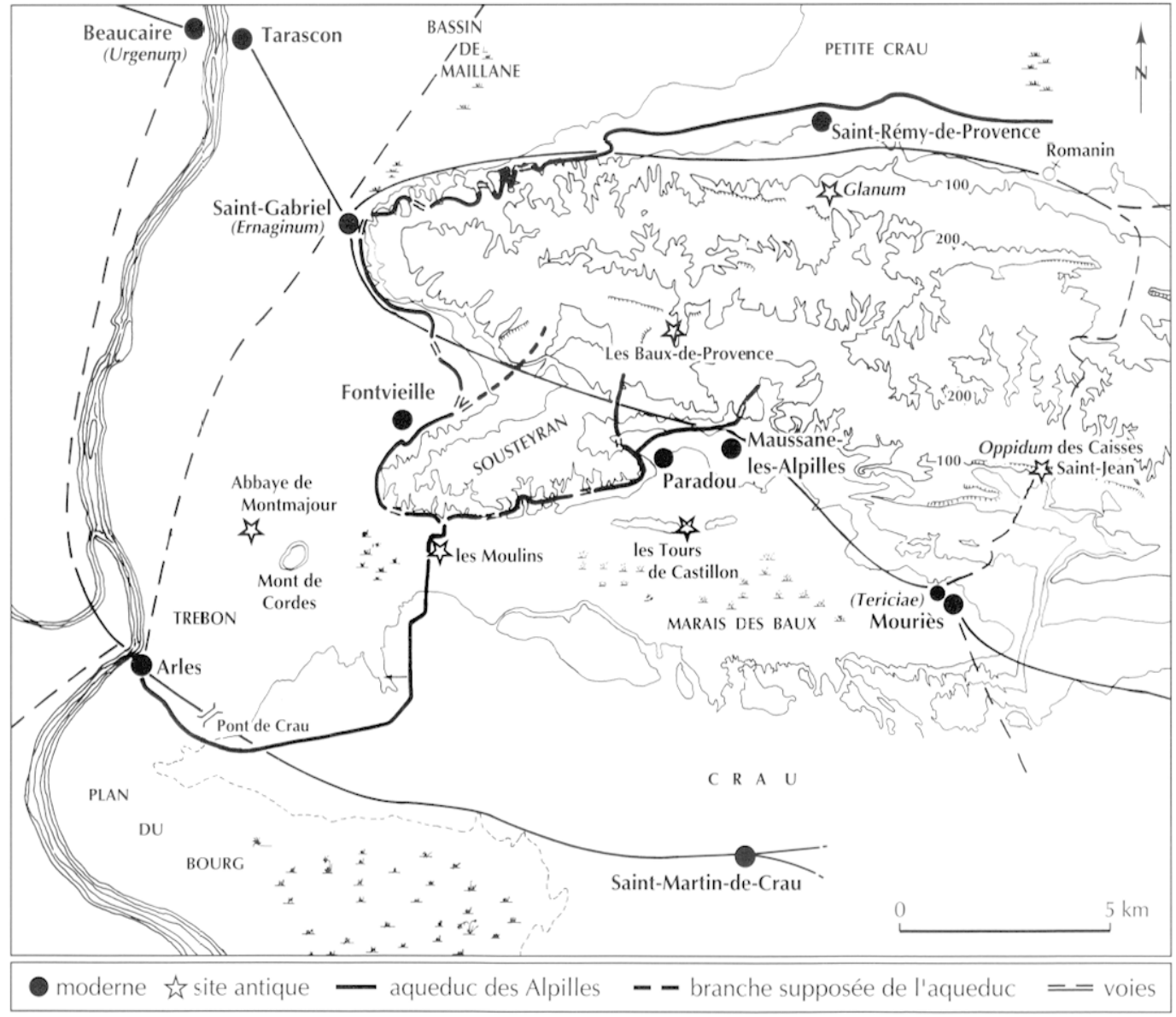

Fig. 79 - Tracé des aqueducs des Alpilles qui alimentaient la colonie romaine d'Arles (dessin M. Borély, CNRS). 
ultérieure, il a été partiellement détruit de façon à laisser à nouveau couler l'eau. Nous le nommerons mur oriental du bras mort ;

- à son extrémité aval (ouest), une autre construction obstruait le débouché de ce canal dans le bassin de convergence. C'est le mur occidental du bras mort. Comme le mur oriental, ce dernier a été ensuite abaissé pour permettre à nouveau la circulation de l'eau.

Ce tronçon présente également, à $1 \mathrm{~m}$ de son débouché aval, un étranglement constitué par deux blocs de pierre, qui reposent sur des concrétions et matériaux détritiques accumulés dans ce bras mort (étranglement du bras mort).

Les travaux sur les concrétions du bassin de convergence du vallon des Arcs et des conduits adjacents ont permis de confirmer et de préciser l'histoire de l'ouvrage et d'apporter des arguments décisifs pour la datation des moulins de Barbegal (Guendon, Villeminot, 1992).

\section{STRUCTURES ET GÉOMÉTRIE DES CONCRÉTIONS DES AQUEDUCS}

À la suite des divers travaux effectués sur les concrétions de plusieurs aqueducs du Midi de la France (Guendon, Villeminot, 1992 ; Guendon, Vaudour, 2000 ; Guendon et al., 2002), nous distinguons, suivant leur position par rapport au canal, les concrétions externes et les concrétions internes.

\section{LES CONCRÉTIONS EXTERNES}

Elles forment des revêtements sur les parois externes de l'ouvrage ou des stalactites et stalagmites sous les arches. Le plus souvent elles mettent en évidence des dysfonctionnements de l'aqueduc, tels que :

- des fuites, liées à un défaut de construction ou à des dégradations de l'ouvrage ;

- des débordements, consécutifs à de fortes crues ou à un développement excessif des concrétions internes ou encore à des défauts d'entretien pouvant entraîner une obstruction du conduit, par exemple.

Elles peuvent également correspondre à des soutirages et dérivations volontaires des eaux de l'aqueduc pour des besoins locaux.

L'étude de l'aqueduc de Nîmes a révélé de nombreux dépôts de fuite plus ou moins importants, mais également plusieurs gros amas externes de tuf en relation avec des dérivations volontaires de l'eau (Guendon, Vaudour, 1986, 2000), ainsi que des surverses ${ }^{74}$ de bassins régulateurs (Fabre et al. dir., 2000). Sur les parties étudiées de l'aqueduc d'Arles, les concrétions externes, peu abondantes, semblent plutôt liées à des fuites. Mais, ces dépôts externes ne pouvant exister que sur des parties construites en élévation, ce qui n'est pas le cas du bassin de convergence de l'aqueduc d'Arles qui constitue l'essentiel du présent travail, nous ne nous étendrons pas plus sur ce sujet.

74. Évacuation par débordement sur la partie supérieure d’un bassin.

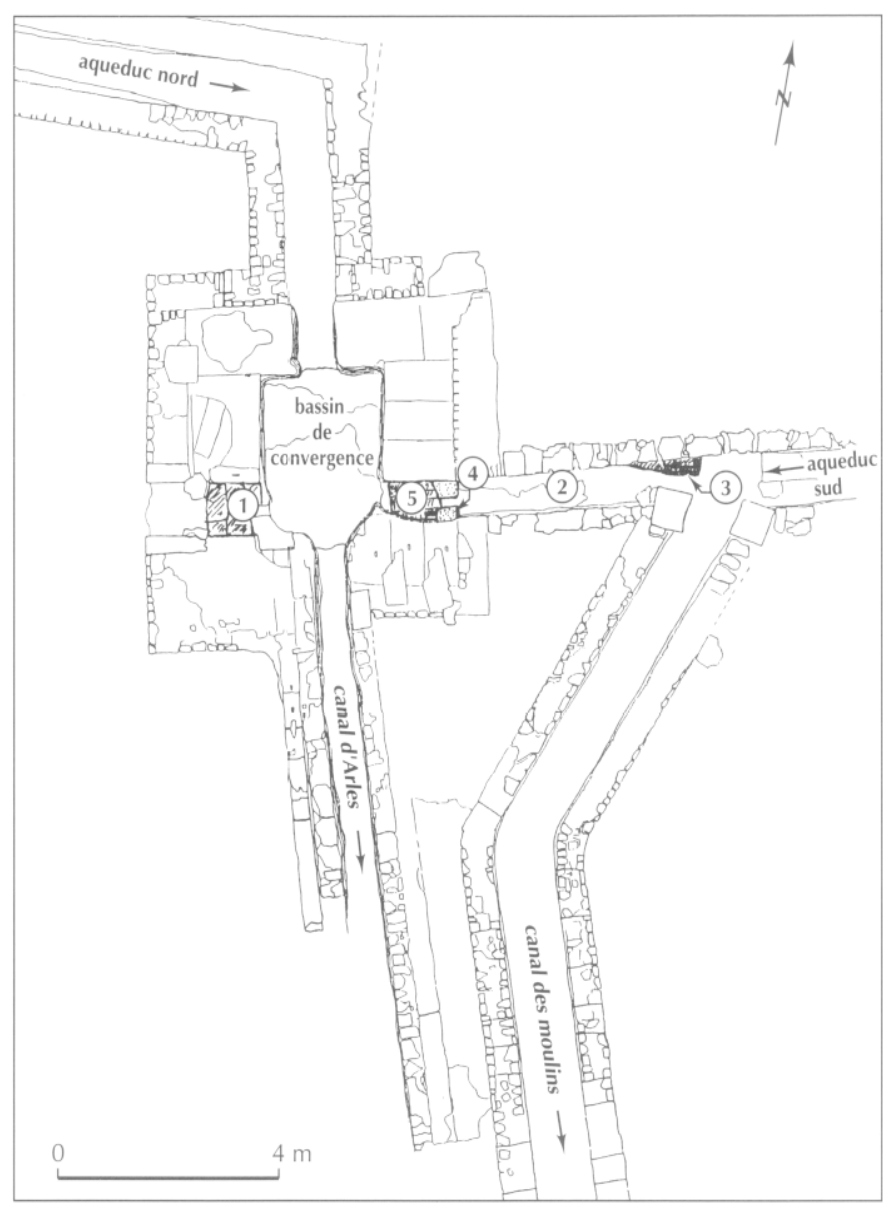

Fig. 80 - Plan du bassin de convergence des aqueducs d'Arles au vallon des Arcs: 1 , ouverture murée occidentale; 2 , bras mort de l'aqueduc sud; 3, vestiges du mur oriental du bras mort;

4, étranglement du bras mort ; 5, mur occidental du bras mort (dessin M. Kleniec, modifié d'après des relevés topographiques de M. Kleniec, A. Carrière, J.-L. Guendon et P. Villeminot, CNRS).

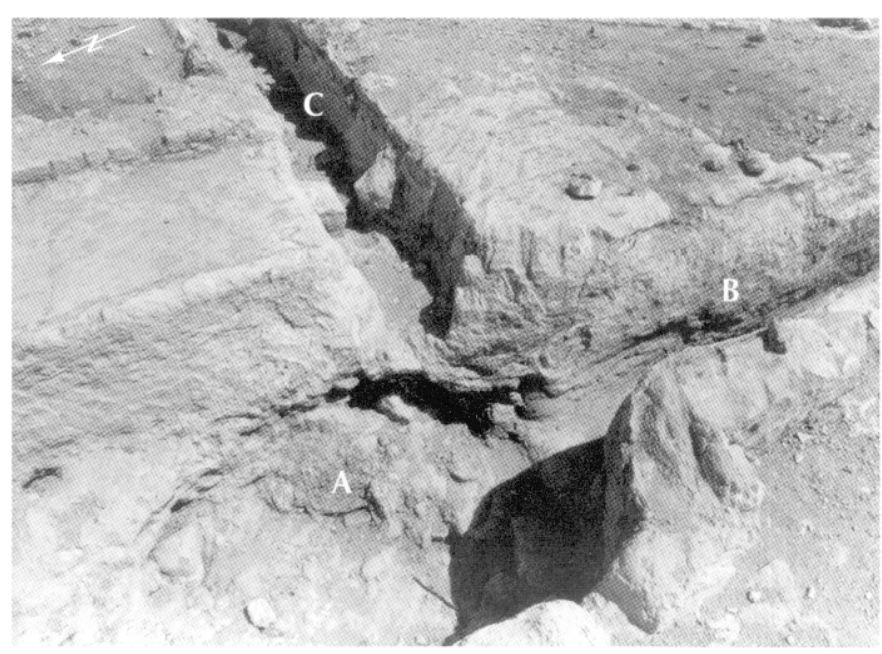

Fig. 81 - Les dépôts concrétionnés dans le bassin de convergence et au niveau du mur occidental du bras mort (voir fig. 80, $n^{0} 5$ et fig. 82B): $A$, bassin de convergence; $B$, aqueduc d'Arles ; $C$, bras mort (cliché P. Foliot, CNRS). 


\section{LES CONCRÉTIONS INTERNES}

Elles sont constituées par l'accumulation régulière, sur les surfaces internes des conduits, de très fins lits (ou lamines) de calcite, plans ou légèrement ondulés, parallèles les uns aux autres. Ces concrétions, déposées par les eaux qui ont circulé dans l'aqueduc, enregistrent différents événements survenant durant le fonctionnement de l'ouvrage. Elles marquent les variations du niveau de l'eau dans les conduits. Elles peuvent donc servir à estimer les paléodébits. Elles enregistrent, en principe, la plupart des événements et perturbations de ce fonctionnement: arrêt des écoulements; variations dans l'origine, la qualité ou le sens de l'écoulement de l'eau ; travaux de réparation et d'entretien. En un point donné, leur épaisseur est théoriquement fonction de la durée du fonctionnement. Cependant, les turbulences, qui accroissent localement la vitesse du concrétionnement, ou, à l'inverse, les interruptions de l'écoulement, les grattages plus ou moins superficiels ou parfois les curages complets des concrétions, qui provoquent des pertes d'informations, imposent une grande prudence dans les interprétations en ce domaine. Ce sont ces concrétions internes qui ont apporté des informations sur les multiples usages du bassin de convergence, sur la chronologie des différents conduits et sur la datation de la meunerie de Barbegal.

\section{LES AUTRES DÉPÔTS INTERNES}

À côté des dépôts concrétionnés il peut exister également des dépôts meubles, qualifiés de détritiques parce qu’ils renferment des éléments fins ou grossiers remaniés. Ils peuvent être superposés aux concrétions ou interstratifiés dans les incrustations. Ce peut être des fragments de roche, des sables ou des argiles introduites accidentellement dans le conduit lors de crues ou depuis les regards, ou lors de dégradations ou effondrements partiels de la voûte et des parois. Ce peut être aussi des débris manufacturés divers: fragments de céramique, de métaux ou de matériaux de l'aqueduc, comme des éclats de taille des blocs, des débris de mortier ou de concrétions résultant de travaux de réparation, d'entretien, de décroûtage ou de dégradation de l'ouvrage. Par rapport aux aqueducs de Nîmes et de Fréjus par exemple, on remarque au vallon des Arcs la présence de nombreux dépôts détritiques internes. Ils remplissent le bras mort à mi-hauteur, couvrent le fond du bassin de convergence et apparaissent dans le canal d'Arles. Ils traduisent les multiples remaniements architecturaux effectués dans ce secteur.

\section{RÉSULTATS DÉDUITS \\ DE L'ANALYSE DES CONCRÉTIONS ET DÉPÔTS DÉTRITIQUES INTERNES}

l.e travail a porté essentiellement sur le site du bassin de convergence et les conduits immédiatement adjacents, où les concrétions sont bien conservées et relativement abondantes : 15 à $20 \mathrm{~cm}$ d'épaisseur en moyenne sur les parois, sauf au début du canal des moulins où clles n'ont que $5 \mathrm{~cm}$. Cependant, le tronçon de l'aqueduc nord, juste à l'amont du bassin, étant mal conservé, nous avons préféré étudier la branche nord plus à l'amont, sur une meilleure section de l'ouvrage en bordure de la route de Fontvieille. Nous avons également préféré étudier les concrétions de l'aqueduc sud en un point éloigné de la dérivation des moulins, la mise en place de ce nouveau conduit ayant vraisemblablement occasionné un curage local de l'ancien canal : juste à l'amont de la dérivation des moulins, le tronçon de l'aqueduc sud présente, en effet, des concrétions très peu épaisses par rapport à celles visibles dans le secteur étudié à $500 \mathrm{~m}$ à l'amont. Sur les ponts de l'aqueduc d'Arles et des moulins, les concrétions internes, trop dégradées, n'ont pas fait l'objet d'observations détaillées. L'apport de l'étude des concrétions est fondamental pour deux raisons : elle permet d'individualiser des séquences et d'en approcher la durée.

\section{LES DIFFÉRENTES GÉNÉRATIONS DE CONCRÉTIONS}

On peut distinguer, dans les concrétions internes du bassin de convergence et des canaux adjacents, cinq grands ensembles concrétionnés correspondant à cinq générations de dépôts (fig. 82). Ils sont tous plus ou moins associés à des couches détritiques. Ils sont séparés par des phases d'entretien et de modification dans le fonctionnement de l'ouvrage.

\section{LE PREMIER ENSEMBLE}

De 10 à $15 \mathrm{~cm}$ d'épaisseur, il n'est intégralement conservé que dans le bras mort de l'aqueduc sud (fig. $82, n^{\circ} 7$ ). Il est constitué de trois couches de concrétions. La première, à structure finement laminée, repose sur un enduit de mortier de tuileau. Un petit niveau détritique la sépare de la deuxième couche, blanchâtre, à surface mamelonnée. Les constituants de ce dépôt détritique sont dans l'ensemble fortement émoussés et la part des débris manufacturés est quasi nulle. Ces caractéristiques laissent supposer une mise en place "naturelle " correspondant à un écoulement soutenu des eaux. Ces deux premières couches de concrétions ne montent qu'entre 37 et $47 \mathrm{~cm}$ de haut sur les parois. En revanche, la troisième s'élève à $90 \mathrm{~cm}$ de haut. Cet ensemble passe sous les murs édifiés aux extrémités du bras mort lors de la construction des moulins. Il a été entaillé pour permettre la mise en place du monolithe qui arme l'angle ouest du départ du canal des moulins. Dans l'aqueduc sud, au niveau du raccord avec ce canal, on retrouve des traces de ces premières concrétions entre l'enduit de mortier de tuileau originel du fond de l'aqueduc sud et celui mis en place par la suite lors de la construction du canal des moulins. Ce premier ensemble est donc antérieur à la construction des moulins.

Dans les autres parties de l'ouvage on retrouve des traces de cet ensemble.

- Quelques lambeaux subsistent sur la moitié supérieure du flanc oriental et sur le flanc occidental du bassin de convergence, en particulier sur l'enduit de mortier de tuileau qui recouvre le mur de bouchage de l'ouverture murée occidentale de ce bassin. Ce dernier point tendrait à démontrer que cette ouverture a été obturée très tôt, sans avoir jamais fonctionné. Fn effet, sous le mur d'obturation, les parois de cette ouverture ne présentent pas de concrétions. Dans le reste du bassin, et en 


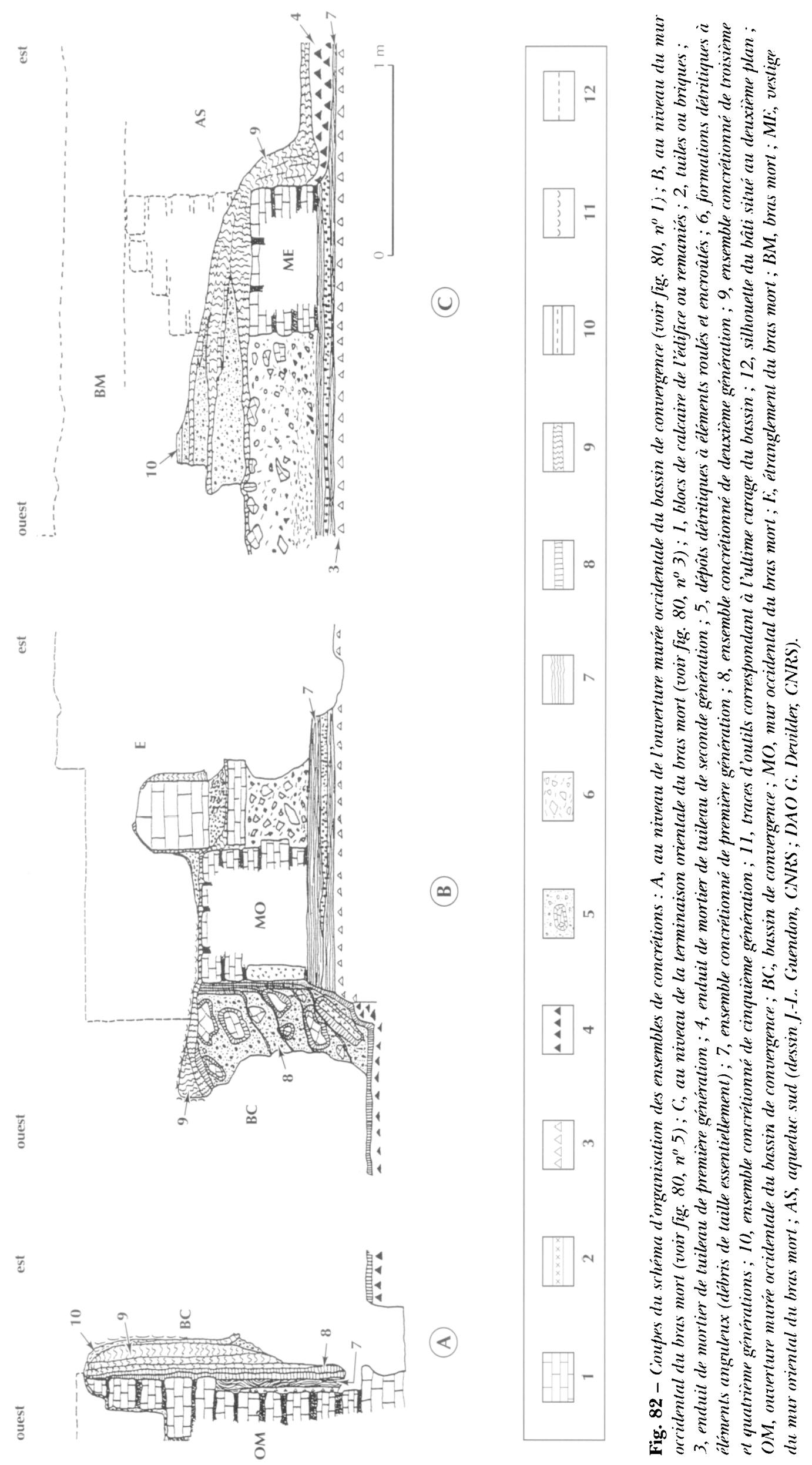




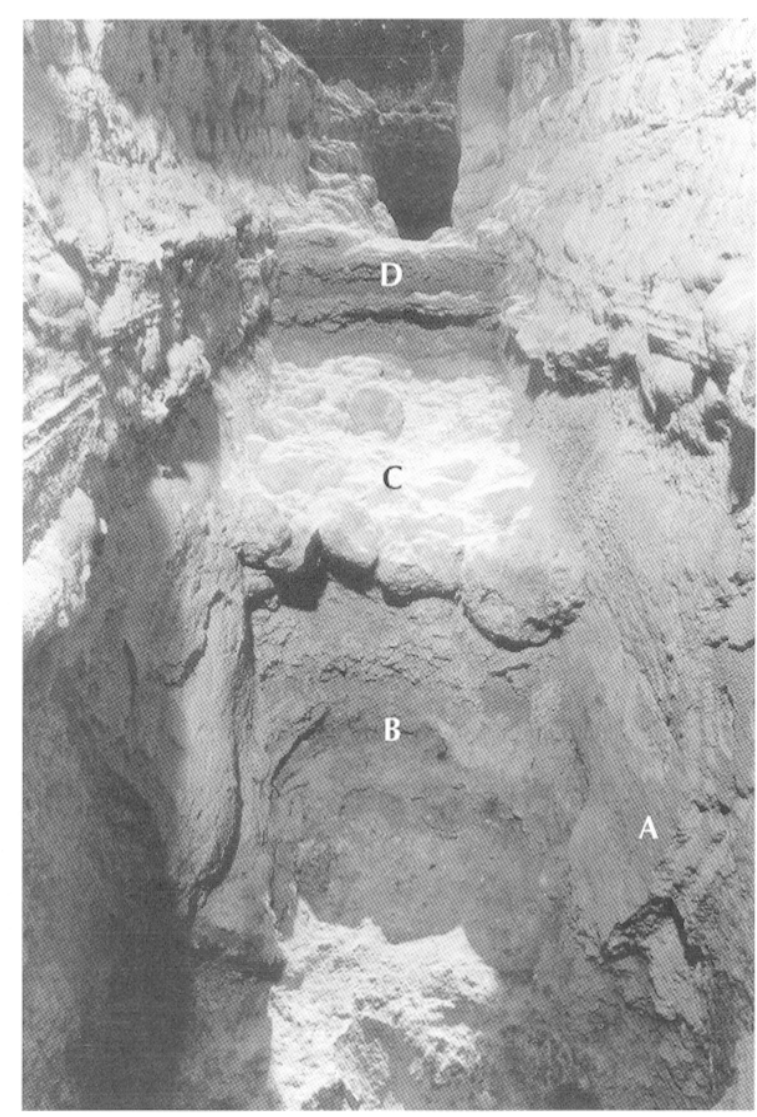

Fig. 83 - Séquence sédimentaire dans le bras mort (voir fig. 82C) : $A$, ensemble concrétionné de première génération sur le piédroit du conduit; B, remplissage détritique à éléments anguleux et débris manufacturés (voir fig. 82C, $n^{\prime \prime}$ 6); (;, ensemble concrétionné de troisième el quatrième générations; 1 , ensemble concrétionné de cinquième génération (cliché P. Foliot, CNRS).

particulier sur le fond, ces premières couches semblent absentes; elles auraient été curées lors des travaux de réfection du bassin.

- Cet ensemble apparaît également dans le canal d'Arles, où il est entaillé par deux encoches visibles à $30 \mathrm{~cm}$ du départ de ce conduit.

\section{LE DEUXIÈME ENSEMBLE}

Il constitue la majeure partie des concrétions et des dépôts détritiques visibles dans le bassin de convergence. Il recourre en particulier la face ouest du mur qui ferme l'extrémité aval du bras mort (fig. $82, n^{\circ} 8$ ).

Cet ensemble est donc postéricur à la séparation des branches nord et sud, donc postérieur à la construction des moulins. Il semble remonter au début du $\mathrm{II}^{\circ}$ s. apr. J.-C.. puisqu il repose directement sur l'enduit de mortier de tuileau contenant un as de Trajan et qu il renferme des monnaies de la même époque.

On retrouve également cet ensemble de concrétions dans le canal d'Arles, où, comme le premier ensemble, il est antéricur aux deux encoches visibles au départ de ce conduit ; mais il n'a pas été observé dans le bras mort, ce qui paraît évident puisque ce tronçon était alors bouché. Dans ce même bras mort, les matériaux détritiques compris entre les concrétions du premier ensemble et celles du troisième seraient contemporains de cette période (fig. 82 et 83). L'essentiel de ces matériaux, surtout dans la partie supérieure du dépôt, est très riche en débris manufacturés centimétriques, peu usés, et en éclats de taille parfois décimétriques, généralement anguleux. On note, d'autre part, la présence de nombreux fragments d'encroûtement autour de racines et une malacofaune abondante. Ce remplissage détritique correspondrait à un dépôt volontaire dans la mesure où la fermeture momentanée du bras mort a nécessairement provoqué des travaux générateurs de débris de taille. Dès lors que le tronçon devenait inutile, on conçoit aisément que ces déchets encombrants furent entreposés à proximité des travaux, en l'occurrence dans le conduit désaffecté.

I ces premières couches de concrétions du canal des moulins seraient également de cette époque, tout en étant d'origine différente, car déposées par les eaux drainées par la branche sud.

\section{LES TROISIÈME ET QUATRIÈME ENSEMBLES}

Ils débutent par une couche caractéristique dure, brune, bien cristallisée, à structure laminée, que l'on peut suivre facilement, et qui a résisté aux curages ultérieurs (fig. 82, n9). Les couches supérieures, plus poreuses, peuvent atteindre localement une vingtaine de centimètres d'épaisseur, mais elles semblent fortement entamées par ces curages ultérieurs (fig. $82, \mathrm{n}^{\circ} 11$ ), anté- et post-cinquième ensemble.

Ces dépôts sont postérieurs à la réouverture du bras mort ; ils recouvrent les restes des murs est et ouest qui obturaient ce tronçon. En effet, pour permettre de nouveau les échanges entre les branches nord et sud, mais cette fois avec un écoulement ouest-est, ces murs ont été entaillés et abaissés. Initialement, le mur ouest avait au moins $15 \mathrm{~cm}$ de plus et celui de l'est a été détruit sur plus de $60 \mathrm{~cm}$ de haut dans sa partie sud. Plutôt qu'une réouverture complète du bras mort, ces entailles réduites des murs de bouchage avaient sans doute pour objet de réguler les débits et de n'envoyer, vers les moulins, qu'une faible partie des eaux de la branche nord. Un deuxième système de régulation du débit a d'ailleurs été installé, peu après, sous la forme de deux blocs de calcaire molassique (étranglement du bras mort). Mais cette installation ne semble pas accompagner immédiatement l'ouverture du bras mort, car les premières lamines de concrétions passent sous ces blocs. On peut ainsi individualiser ces premières couches pour déterminer le troisième ensemble. À l'inverse, les couches suivantes recouvrent ces mêmes blocs. C'est l'ensemble quatre. Dans le bras mort, des épisodes détritiques s'intercalent entre ces concrétions. Ces dépôts meubles renferment de nombreux débris manufacturés de très petite taille et souvent usés. Ils se distinguent des précédents épisodes détritiques par l'abondance de quartz roses, émoussés et luisants, identiques à ceux trouvés dans l'Éocène, dont les seuls affleurements connus dans la région se situent au sud-est de Saint-Rémy-deProvence, sur le flanc nord des $\Lambda$ lpilles, à proximité du tracé de l'aqueduc nord. Les planchers concrétionnés qui alternent 
avec ces dépôts détritiques viennent se confondre au-dessus du mur oriental et présentent une terminaison de type cascadant au niveau de leur raccord avec le canal des moulins. Cette disposition des couches détermine un écoulement vers l'est. Cela s'accorde parfaitement avec la présence des quartz roses dans les épisodes détritiques associés; présence qui indique une alimentation venant de la branche nord de l'aqueduc.

Ces ensembles envahissent les deux encoches visibles au départ du canal d'Arles ; ils sont donc postérieurs à leur creusement. Ces encoches n'auraient donc eu qu'un usage bref entre le dépôt de l'ensemble deux, entaillé par le creusement des encoches, et l'ensemble trois-quatre, qui, en les remplissant, rend leur utilisation impossible. Le rôle de ces entailles reste hypothétique. Servaient-elles d'ancrage pour un système de régulation du débit par vannage ? Mais ces encoches ne se prolongent pas vers le bas des parois et aucune trace particulière n'apparaît entre les ensembles deux et trois-quatre à cet endroit.

\section{LE CINQUIÈME ENSEMBLE}

Il est postérieur au creusement du petit déversoir occidental du bassin de convergence, visible au-dessus de l'ouverture murée occidentale (fig. 82, $\mathrm{n}^{\circ} 10$ ). Ce déversoir apparaît ainsi très tardif, aucune trace d'ouverture antérieure n'ayant été décelée. Lne activité de curage des concrétions semble, cette fois encore, accompagner ces nouveaux aménagements hydrauliques, en particulier au départ du canal d'Arles où des traces d'outils séparent cet ensemble de l'ensemble précédent. Il est vrai qu'en ce point, favorable aux remous, le concrétionnement très actif (30 à $40 \mathrm{~cm}$ d'épaisseur visible actuellement) devait nécessiter un entretien particulier.

\section{HISTOIRE DU FONCTIONNEMENT DE L'OUVRAGE ET COMMENTAIRES}

À travers les connaissances antérieures et les premiers résultats de l'étude des concrétions, le fonctionnement du bassin de convergence du site du vallon des Arcs peut se résumer de la façon suivante (fig. 84 et 85 ).

\section{PREMIÈRE PHASE}

- Construction du bassin.

- Bouchage de l'ouverture occidentale.

Le système fonctionne alors en régime de convergence des eaux des branches nord et sud pour alimenter Arles (fig. 84A), et cela pendant une durée non négligeable puisque sur le fond du bras mort les concrétions du premier ensemble ont entre 10 et $15 \mathrm{~cm}$ d'épaisseur. En utilisant la vitesse moyenne de concrétionnement de $2 \mathrm{~mm}$ par an estimée sur l'aqueduc de Fréjus (Guendon et al., 2002), on obtiendrait une durée de 50 à 75 ans pour cette première phase.

\section{DEUXIÈME PHASE}

- Construction de la meunerie.

- Curage partiel et réfection de l'enduit de mortier de tuileau du bassin de convergence.
- Séparation des branches sud et nord.

- Construction du canal et du pont des moulins de Barbegal.

Le système fonctionne cette fois en régime de séparation des eaux des branches nord et sud. Avec les $12 \mathrm{~cm}$ d'épaisseur de concrétions du deuxième ensemble, vers le haut des parois du bassin, on pourrait estimer la durée de cette deuxième phase à une soixantaine d'années. La volonté de séparer les alimentations (la branche sud alimentant la meunerie et la branche nord, Arles) se marque nettement par la fermeture du bras mort et la construction du pont des moulins de Barbegal. L'alimentation pour une ville ne correspondait pas aux mêmes exigences de qualité des eaux, que pour un établissement industriel. Ainsi apparaissait la possibilité d'augmenter le débit de la branche sud, au détriment de la qualité, en captant des eaux d'origines diverses (rivières ?). L'aqueduc sud, à l'aval du bassin de la Burlande, présente, en effet, au-dessus d'un premier ensemble de concrétions, des dépôts riches en argiles et limons (J. Vaudour, communication orale). Or, par sa structure et son épaisseur, ce premier ensemble de l'aqueduc sud à la Burlande est strictement comparable au premier ensemble du bras mort de l'aqueduc sud au bassin de convergence. Les premières couches de la Burlande seraient donc également antérieures aux moulins et correspondraient à une circulation d'eaux claires pour Arles. En revanche, les dépôts suivants, plus terrigènes, correspondraient à la période d'alimentation des moulins par des eaux plus turbides.

Côté branche nord et bassin, on constate que cette période correspond à l'accumulation du matériel détritique grossier visible dans le bassin de convergence (fig. 84B). Ces apports pourraient résulter de crues et/ou de dégradations sur la branche nord.

\section{TROISIÈME ET QUATRIÈME PHASES}

- Réouverture partielle du bras mort (phase trois).

- Mise en place d'un étranglement sur ce tronçon d'aqueduc (phase quatre).

Durant ces deux phases, le bassin fonctionne en divergence pour les eaux de la branche nord (fig. $84 \mathrm{C}$ et D) : une partie de ces eaux continue d'alimenter Arles, tandis qu'une autre partie va s'ajouter, via l'ex-bras mort, aux eaux de la branche sud pour alimenter les moulins.

Les deux encoches visibles au départ du canal d'Arles posent des problèmes. Correspondent-elles à un système de régulation ou d'obturation du canal d'Arles (pour faciliter la réparation et la reconstruction du pont de l'aqueduc d'Arles qui enjambe le vallon des Arcs, par exemple) ? Le système a-t-il fonctionné ? Si c'est le cas, ce serait entre les ensembles deux et trois-quatre, pour une courte durée et sans laisser apparemment d'autres traces que ces deux encoches.

\section{CINQUIÈME PHASE}

- Ouverture du déversoir ouest du bassin.

- Curage partiel des concrétions.

Le bassin fonctionne toujours en divergence: une partie des eaux du nord pour Arles, une autre partie pour les moulins. 
La période de fonctionnement en divergence du bassin $\left(3^{\mathrm{e}}\right.$, $4^{\mathrm{e}}$ et $5^{\mathrm{e}}$ phases) serait au total relativement longue : sur le haut des parois du bassin, l'épaisseur des ensembles 3,4 et 5 dépasse $15 \mathrm{~cm}$, et cela malgré deux curages d'une partie des concré- tions, l'un intermédiaire et l'autre postérieur. Cette période aurait donc duré bien plus de 75 ans.

Les divers épisodes de curage du bassin, avec des grattages plus ou moins importants des concrétions, se situeraient entre

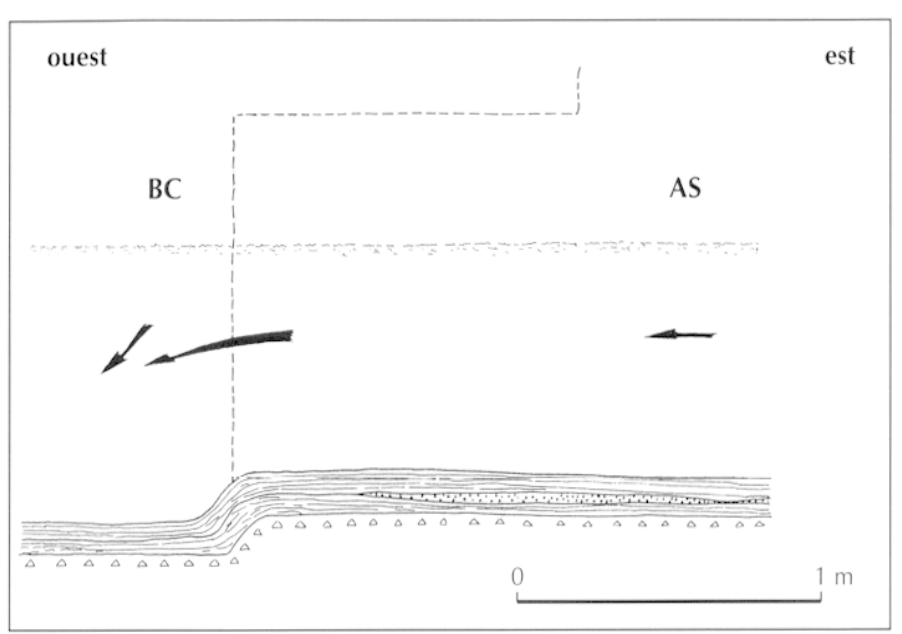

(A)

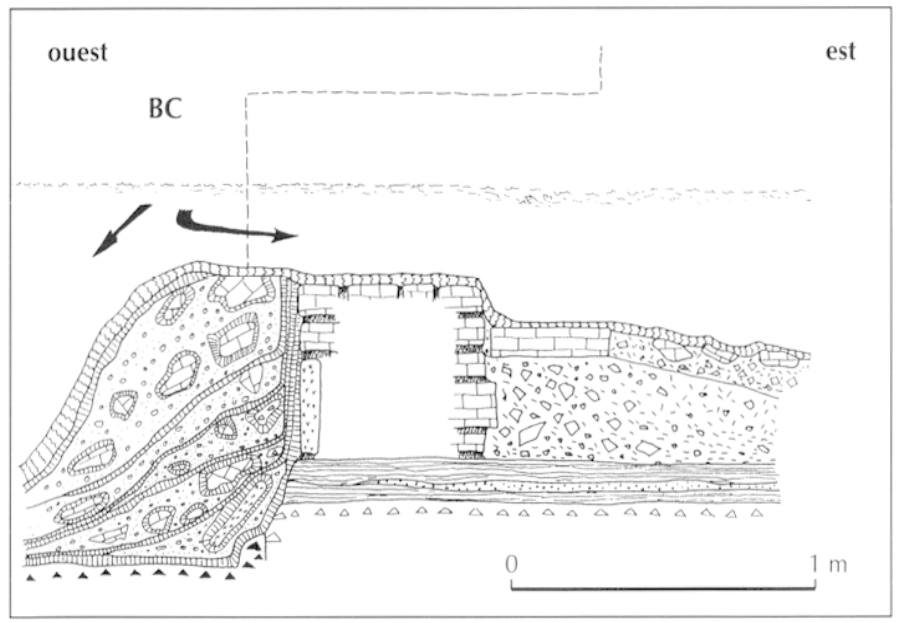

(C)

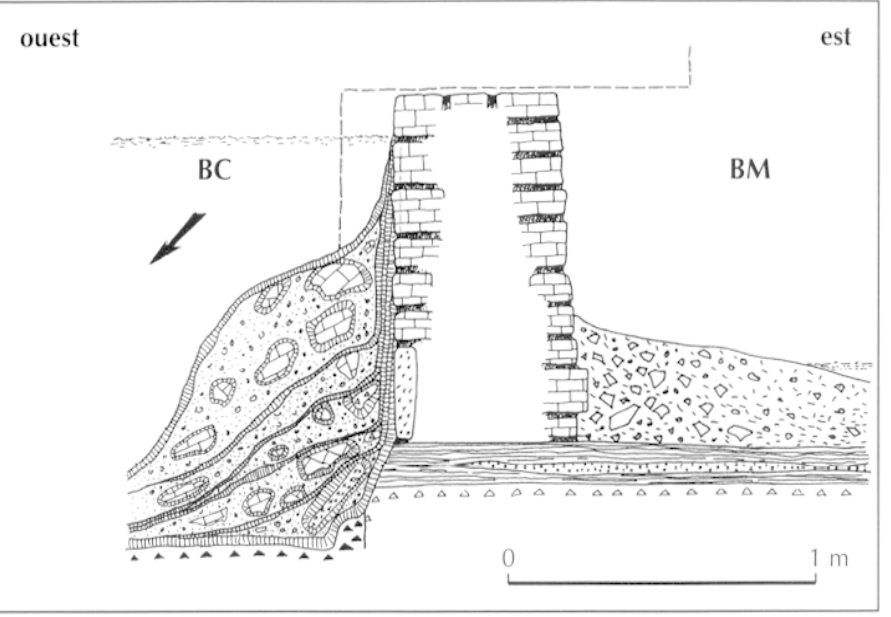

(B)

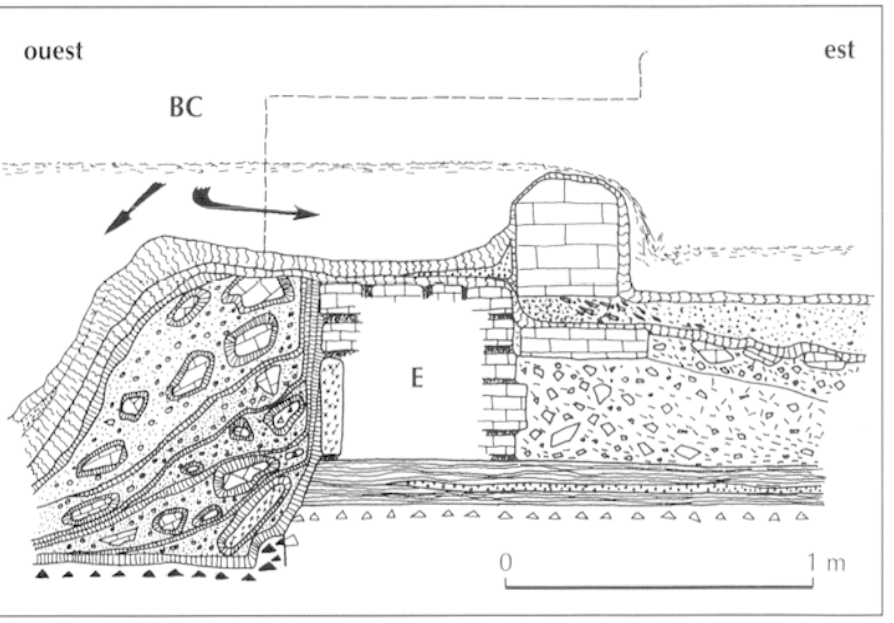

(D)

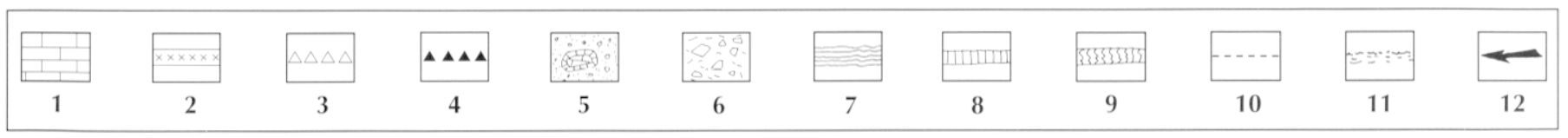

Fig. 84 - Coupes de l'éoolution dans le temps des dépôts et du fonctionnement de l'aqueduc au niveau du mur occidental du bras mort (voir fig. 8(), $\left.n^{\circ} 5\right):$ A, première phase, convergence des aqueducs nord el sud, dépôt des concrétions de première génération ( $I^{p r}$ s. ?); B, deuxième phase, restauration du bassin de convergence el séparation des aqueducs nord et sud (II's. ?), dépôt des concrétions de deuxième génération ; (', troisième phase, abaissement du mur occidental du bras mort et réutilisation de l'ex-bras mort pour dériver une partie des eaux de l'aqueduc nord vers les moulins de Barbegal via l'aqueduc sud, dépôt des concrétions de troisième génération ; 1 , quatrième phase, édification de l'étranglement du bras mort (seuil pour réguler le débit ?) (voir fig. 80, $n^{\prime \prime} 4$ ), dépôt des concrétions de quatrième génération ; 1 , blocs de calcaire de l'édifice ou remaniés ; 2, tuiles ou briques; 3, enduit de mortier de tuileau de première génération; 4, enduit de mortier de huilean de deuxième génération; 5 , défôts détritiques à éléments roulés el encroûtés; 6, formations détritiques à éléments anguleux (débris de taille essentiellement); 7, ensemble concrétionné de première génération ; 8, ensemble concrétionné de deuxième génération ; 9, ensemble concrétionné de troisième et quatrième générations ; 10, silhouette du bâti situé au second plan ; 11, niveau moyen de l'eau dans l'ouvrage; 12, sens de l'écoulement des eaux; BC; bassin de convergence; AS, aqueduc sud ; BM, bras mort; L, étranglement du bras mort (dessin J.-L. (iuendon, CNRS; DAO (s. Devilder; (XNRS). 

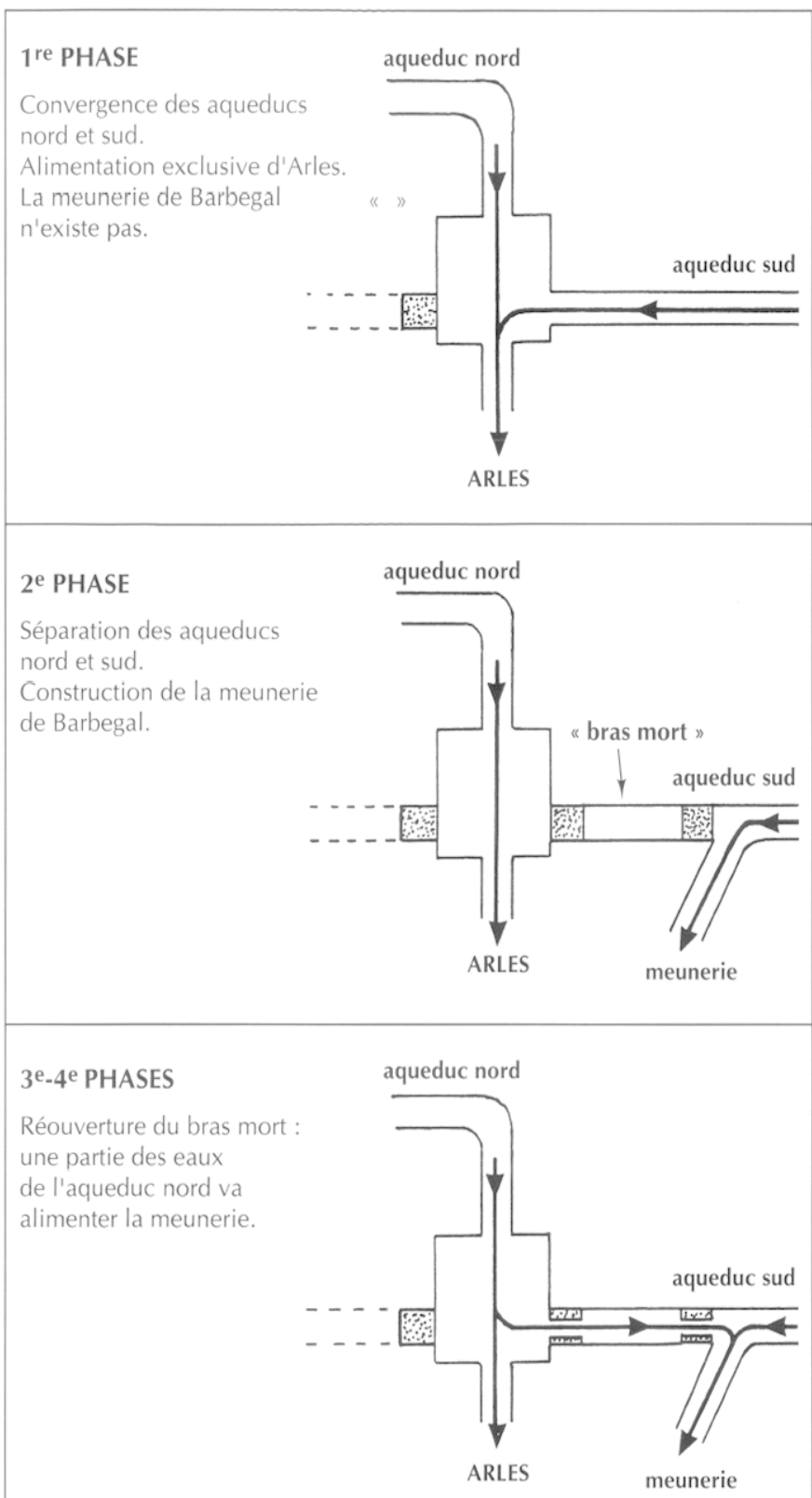

5 PHASE

Ouverture d'un déversoir sur le mur ouest du bassin de convergence.

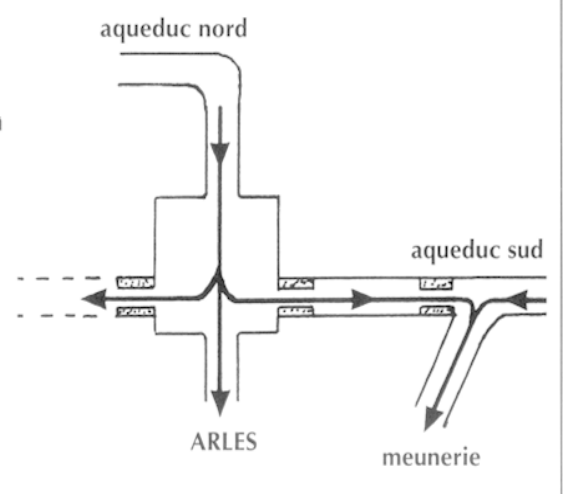

Fig. 85 - Plans schématiques des principales phases $d u$ fonctionnement du bassin de convergence des aqueducs d'Arles au. vallon des Arcs (dessin J.-L. Guendon, CNRS; DAO G. Devilder; CNRS). les ensembles un et deux, puis entre les ensembles trois-quatre et cinq et à la fin du fonctionnement. L'aqueduc a semble-t-il cessé de fonctionner peu après l'ultime curage des concrétions, car les traces d'outils sur cette surface curée ne semblent pas être recouvertes par de nouvelles concrétions.

Ces observations de terrain ont été confirmées par des analyses chimiques de concrétions. Deux éléments-traces sont particulièrement significatifs: le strontium (Sr) et le baryum $(\mathrm{Ba})$. Les dosages de ces éléments dans diverses couches de concrétions prélevées en différents points de la branche nord (au total vingt-quatre analyses : à la Roche percée et au vallon de la Baume, à l'amont du mas d'Auge ; sur la route de Fontvieille, quelques centaines de mètres à l'amont du vallon des Arcs et à l'entrée du bassin de convergence) présentent des taux de strontium variant entre 490 et 295 ppm (parties par million), soit $367 \mathrm{ppm}$ en moyenne, et des taux de baryum variant entre 72 et $43 \mathrm{ppm}$ (moyenne : $55 \mathrm{ppm}$ ). Sur la branche sud, treize analyses ont été effectuées : à l'entrée et à la sortie du bassin de la Burlande, près du Paradou, et $500 \mathrm{~m}$ à l'amont $\mathrm{du}$ vallon des Arcs. Les teneurs en strontium y sont dans l'ensemble plus faibles, entre 394 et 223 ppm, et malgré deux valeurs supérieures à 448 et 513 ppm, localisées à l'entrée du bassin de la Burlande, la moyenne pour cet élément reste sensiblement inférieure à celle observée dans la branche nord $(325 \mathrm{ppm})$. Pour le baryum la différence est plus nette avec des valeurs entre 59 et $22 \mathrm{ppm}$ seulement, et une moyenne de 35 ppm. Dans le bras mort, on retrouve bien pour les concrétions de la première génération (sept analyses) les taux de strontium et de baryum relativement faibles de la branche sud: Sr entre 298 et 263 ppm et Ba entre 23 et 43 ppm. En revanche, les concrétions de troisième et quatrième générations (cinq analyses) présentent de forts taux de strontium et de baryum, comme dans la branche nord: Sr entre 433 et 300 ppm et Ba entre 58 et $49 \mathrm{ppm}$. Cela confirme, qu'à cette période, les eaux de la branche nord transitaient bien par l'ex-bras mort pour venir s'ajouter aux eaux de la branche sud et alimenter les moulins. Pour confirmation, les deux analyses effectuées sur les concrétions de deuxième génération dans le bassin de convergence présentent bien les hautes valeurs de la branche nord (Sr : 381 et 371 ppm; Ba : 57 et 53 ppm).

\section{CONTRIBUTION À LA DATATION DES MOULINS DE BARBEGAL}

Des datations absolues ou approchées ont été fournies par les ensembles numismatiques et céramologiques constituant les contextes archéologiques mis au jour dans le bassin et dans l'exutoire des moulins (Leveau et al., 2000).

L'étude de la composition des enduits intérieurs des mortiers de tuileau réalisée par P. Girard en 1991 avait permis de distinguer deux familles qui pouvaient correspondre à deux périodes de travaux (Girard in Leveau, 1995). Les mortiers situés sous les concrétions de première génération, donc les plus anciens, comme dans le bras mort ou sur l'ouverture murée occidentale du bassin de convergence, se distinguaient des autres par un taux de chaux beaucoup plus faible. Ce premier enduit, contemporain de la construction de l'ourrage, ainsi que la première génération de concrétion 
le recouvrant, ont été ensuite localement enlevés, en particulier dans le fond du bassin, pour permettre la réfection de ce dernier. Les autres mortiers, revêtus directement par les concrétions de deuxième génération, donc plus récents, présentaient de fortes similitudes entre eux. Ils correspondaient notamment à l'enduit de réfection du fond du bassin de convergence ainsi qu'à celui mis en place lors de la construction du canal des moulins.

L'hypothèse d'une restauration du bassin de convergence se trouvait ainsi confirmée et datée : les concrétions qui recouvrent le fond du bassin et renferment les monnaies du $\mathrm{II}^{\mathrm{e}} \mathrm{s}$. apr. J.-C. sont celles de deuxième génération (Leveau et al., 2000 , p. 415-416). Les restes de concrétions de première génération retrouvés en plusieurs points, en particulier dans le bras mort, prouvaient l'existence et le fonctionnement de l'ouvrage avant le début du $\mathrm{II}^{\mathrm{e}} \mathrm{s}$. apr. J.-C.

L'étude des incrustations calcaires a ainsi permis d'utiliser clairement l'argument numismatique pour dater la construction de la meunerie de Barbegal. Les monnaies ne sont en effet utilisables qu'à la faveur d'une stratigraphie relative, mais précise et sûre, établie par les générations de concrétions. On a pu voir que celles de première génération sont antérieures à la fermeture du bras mort et à la mise en place des monolithes qui arment le départ du canal des moulins. Elles sont donc antérieures à la construction de la meunerie. On a pu voir également que celles de deuxième génération, datées par les monnaies du début du $\mathrm{II}^{\mathrm{e}} \mathrm{s}$. apr. J.-C., se déposent après la séparation des eaux du nord de celles du sud, par fermeture du bras mort. Ces concrétions se déposent donc après l'ouverture du canal des moulins, la séparation des branches nord et sud étant la conséquence de cette volonté d'utiliser les eaux du sud pour autre chose que pour alimenter la ville d'Arles.

L'évaluation de la durée du dépôt des concrétions de première génération, une soixantaine d'années, suggère que la construction de l'aqueduc d'Arles remonterait non au règne d'Auguste, mais au milieu du $\mathrm{I}^{\text {er }}$ s. apr. J.-C. Il serait donc contemporain de celui de Nîmes. P. Leveau a proposé de dater au plus tard des années 260 un événement hydraulique survenu dans l'exutoire occidental des moulins (Leveau et al., 2000, p. 422) ; cet événement correspondrait à la fin de l'utilisation industrielle des moulins de Barbegal. Nous avons suggéré une durée de fonctionnement du bassin en divergence d'au moins trois quarts de siècle et probablement plus. Compte tenu de l'incertitude d'un terminus fondé sur une monnaie dont la lecture est incertaine et sur un lot de céramique dont ce serait la date la plus basse, l'évaluation de la durée de dépôt de ces concrétions invite à remonter sensiblement la date d'arrêt du fonctionnement industriel du site de Barbegal.

$$
*
$$

La multiplicité des transformations enregistrées par cet ouvrage hydraulique peut surprendre dans un premier temps. En fait, cette histoire s'étalerait sur plusieurs siècles, plus de deux siècles d'après les calculs très relatifs fournis par l'épaisseur des concrétions, quatre cents ans d'après les estimations archéologiques. Ce qui donnerait, si l'on retient cette dernière estimation, un événement majeur par siècle en moyenne: construction initiale de l'aqueduc, édification des moulins, réouverture du bras mort, réfection du pont-aqueduc d'Arles. Cependant, on s'aperçoit que chaque ensemble de concrétions recouvre une multitude d'événements et que l'histoire de cet ouvrage est sans doute encore plus complexe qu'il n'y paraît. Par exemple :

- le premier ensemble de concrétions est constitué de trois couches, la dernière montant brutalement beaucoup plus haut que les précédentes. Pourquoi ? À quelles modifications du débit, à quels travaux ou entretiens correspond ce changement brutal?

- les $3^{\mathrm{e}}, 4^{\mathrm{e}}$ et $5^{\mathrm{e}}$ ensembles sont également complexes dans le détail. Premièrement, plusieurs fermetures temporaires du bras mort sont probables au niveau de l'étranglement. Sur les parois du bras mort, entre cet étranglement et la dérivation des moulins, des traces d'un niveau d'eau stagnante sont en effet visibles. Elles se seraient développées entre le dépôt de l'ensemble trois-quatre et celui de l'ensemble cinq, ou au cours de l'ensemble cinq. Deuxièmement, toujours dans le bras mort, deux niveaux détritiques interrompent le concrétionnement de l'ensemble quatre. Quels événements traduisent ces épisodes?

Tous les problèmes ne sont donc pas résolus. L'étude détaillée des concrétions a néanmoins permis, ici comme sur les aqueducs de Nîmes et de Fréjus (Guendon, Vaudour, 2000 ; Guendon et al., 2002), de préciser l'histoire des différents aménagements de cette partie de l'aqueduc antique d'Arles et a fourni des arguments décisifs pour dater la construction des moulins de Barbegal. 